\title{
Las causas del auge y la declinación del Sistema Interamericano de Seguridad: una perspectiva latinoamericana*
}

\section{INTRODUCGIÓN}

El Sistema Interamericano de Seguridad se encuentra en un estado de crisis. Este diagnóstico es ampliamente compartido en razón del papel insignificante que desempeñó dicho sistema (si es que efectivamente le cupo algún papel) en el tratamiento de los graves problemas de seguridad que han amenazado al hemisferio en. los últimos años, tales como la guerra de las Malvinas entre Gran Bretaña y Argentina, el conflicto centroamericano, la invasión de Grenada por parte de Estados Unidos, los problemas limitrofes entre Chile $Y$ Argentina o entre Ecuador y Perú y, más recientemente, la crisis de lo. deuda externa latinoamericana. Sin embargo, el colapso actual del Sistema Interamericano de Seguridad tiene un largo historial; el concepto y las instituciones de seguridad del hemisferio han ido decayendo en cuanto a su prestigio, credibilidad y efectividad por lo menos durante las dos últimas décadas.

Este trabajo tiene por objeto identificar las principales causas del colapso del sistema, más que intentar una descripción detallada de su ascenso Y posterior decadencia. En última instancia, las explicaciones del fracaso del esquema de seguridad hemisférica son relativamente simples: primero, el sistema se basó en premisas falsas como la "noción de hemisferio Occidental" y la supuesta igualdad entre los estados miembros; segundo, los Estados Unidos y Latinoamérica tenían expectativas muy divergentes en relación a esta alianza, lo que más tarde derivó en una sensación de desencanto y amargura cuando las esperanzas iniciales de una de las partes (América Latina) no se cumplieron; y, tercero, los cambios que tuvieron lugar en el contexto internacional en las décadas del sesenta y del setenta, se

*Trabajo presentado en la Primera Conferencia del "Proyecto Interamericano de Seguridad Colectiva" de la World Peace Foundation, Boston, 4 a 6 de mayo 
convirtieron en un factor adicional de desintegración del sistema. Estos tres conjuntos de factores convergen, finalmente, hacia una contradicción central: el sistema proporcionaba seguridad para los Estados Unidos, pero producía inseguridad para América Latina.

En las páginas siguientes, desarrollaremos las ideas precedentes, para concluir con algunas notas sobre una visión que tienen muchos latinoamericanos de lo que podrían ser las bases para un sistema alternativo de seguridad colectiva.

\section{LAS FALLAS INIGIALES DEL SISTEMA: FALSAS PREMISAS $Y$ EXPECXATIYAS DIVERGENTES}

Al concluir la Segunda Guerra Mundial, Estados Unidos emergió como el poder hegemónico mundial indiscutible y elaboró una compleja red de tratados, normas y prácticas de seguridad con el fin de asegurar su posición de dominio: esta red de seguridad se consideró de vital importancia, particularmente en vista del creciente desafío soviético.

La relación estratégico-política entre los Estados Unidos y América Latina quedó sellada por la creación del Tratado Interamericano de Asistencia Recíproca (TIAR) de Río en 1947; la aprobación de la Carta de la Organización de Estados Americanos (OEA) en 1948; y el ahora casi olvidado Pacto de Bogotá en 1948 que, se suponía, constituiría un marco efectivo para la resolución pacífica de las controversias en el hemisferio. Ádemás, Washington promovió Ia materialización de un verdadero "sistema militar interamericano", como lo ha llamado Jack Ghild, mediante la implementación de progxamas de asistencia en seguridad, maniobras militares conjuntas y ejercicios combinados, y mediante la creación de varias instituciones militares de alcance hemisférico 1 .

El concepto de "seguridad colectiva" se incorporó específicamente como un componente clave del naciente sistema interamericano. Con el establecimiento del TIAR, se entendió que Estados Unidos saldría en defensa del hemisferio en el caso de un ataque extracontimental, mientra que la oEA y el Pacto de Bogotá debian ser los mecanismos esenciales para la. mediación y solución. pacífica de los conflictos regionales,

El sistema interamericano se apoyó en dos principios centrales, que muy pronto probarían ser sólo "mitos": primero, la idea del

"Ver Jack Child, Unequal Alliance: the Inter-American Military System, 19387978 (Boulder: Westview 1980). Para una critica de este libro, ver Heraldo Mufioz, "Beyond the Malvinas Crisis: Perspectives on inter-american relations". Latin American Research Review, No I, 1984, pp. 158-172. 
"hemisferio Occidental" y, segundo, la supuesta igualdad entre los estados miembros del sistema ${ }^{2}$.

La noción de hemisferio occidental tuvo su origen en la premisa de que habia "intereses comunes" entre los Estados Unidos y América Latina. Pero, en realidad, desde que se proclamó la doctrina Monroe en 1823, Washington consideró a América Latina y al Garibe más que nada como un área de influencia exclusiva. Por otro lado, muchos países latinoamericanos tuvieron la esperanza que entre la región y Estados Unidos se desarrollaría una "relación especial", basada en el espíritu panamericanista; pero, hacia fines de la década del 40, un gran número de naciones latinoamericanas ya percibía a Estados Unidos como "una amenaza por derecho propio"3, y creía que quizás una elaborada estructura de seguridad interamericana sería útil para limitar el intervencionismo de Washington dentro del hemisferio. Es claro que América Latina se alineó con Washington a medida que la Guerra Fría se hacía más intensa. Sin embargo, se puede llegar a sostener que ya en ese tiempo, la amenaza real a la soberanía de los países de América Latina provenía más del poderoso vecino del norte que de una potencia extracontinental.

El segundo mito subyacente al sistema interamericano fue Ia supuesta igualdad entre los estados miembros del sistema, reconocida en todos los tratados y declaraciones de principio. Pero, en la práctica, la obvia asimetría en las relaciones entre Estados Unidos y la región emergió rápidamente como una característica central del sistema. De hecho, en Washington se pensaba que la seguridad de Estados Unidos dependía, en buena medida, del ejercicio de una hegemonía firme en la región, y que, por lo tanto, toda conducta independiente por parte de los estados latinoamericanos implicaba un desafío a tal hegemonía que debía ser impedido por todos los medios posibles.

El sistema interamericano también fracasó porque, como puede deducirse de las falsas premisas que acabamos de mencionar, las naciones latinoamericanas tenían expectativas muy distintas de las de Estados Unidos con respecto a la estructura hemisférica emergente. Como ha sostenido Van Klaveren, América Latina confiaba en que el sistema implicarfa: a) un esquema de cooperación económica si- $^{-}$

Ter Alberto van Klaveren, "The United States and the Inter-American System", en Robert Wesson y Heraldo Muñoz (ed.), Latin American Views of U.s. Policy (New York: Praeger, 1986)

'Jack Child, citado por Gabriel Marcella, "La Relación Estratégica entre EE. UU. Y América Latina: Este-Oeste o Norte-Sur", trabajo presentado en la Conferencia sobre "Seguridad Regional y Paz en América Latina", Instituto de Estudios Internacionales, Universidad de Chile, Viña del Mar, noviembre de 1984 , p. 8. 
militar al Plan Marshall, y b) un compromiso de Estados Unidos con el principio de la no intervención como base para una relación interamericana armónica ${ }^{4}$. Muy pronto quedó en claro que América Latina exper:mentaria profundas desilusiones en ambos aspectos.

Ya en la década del 50 se hizo evidente que Washington era renuente a incluir aspectos económicos en la agenda interamericana, y más aún, que no pretendía elevar a América Latina al "status" de socio igualitario según la retórica de los "intereses comunes del hemisférico Occidental", o como resultado de la colaboración de América Latina durante la guerra ${ }^{\sigma}$ y en la construcción del orden internacional de postguerra.

Desde su inicio, Estados Unidos utilizó el sistema interamericano para lograr resoluciones que estuvieran de acuerdo con sus objetivos estratégicos: desde una condena global al comunismo internacional, hasta una declaración en favor de la participación de Washington en la guerra de Corea. Pero, cuando la maquinaria de seguridad hemisférica resultaba demasiado lenta o ineficaz para enfrentar lo que Washington definía como un desafío a sus intereses, simplemente actuaba en forma unilateral. En 1954, Estados Unidos intervino en Guatemala primero y després acudió a la OEA; en esta organización se limitó exclusivamente a obtener un sello de aprobación para una política que ya estaba decidida. En la invasión de Bahía Cochinos en 1961 se observó una lógica similar de acción unilateral. Del mismo modo, la invasión de República Dominicana en 1965 también estuvo apoyada por una resolución de la oea y, lo que es más importante, por la creación de una Fuerza Interamericana de $\mathrm{Paz}$ para esa ocasión, a pesar de la fuerte oposición de países latinoamericanos claves como Chile, México, Perú y Uruguay. Así, como señala Jerome Slater, si bien Estados Unidos legitimizó sus políticas unilaterales, lo hizo al costo de deslegitimizar la OEA y el concepto de seguridad colectiva ${ }^{6}$.

A mediados de la década del 60, se hizo evidente que el sistema interamericano se estaba deteriorando rápidamente, en tanto se desvanecían las esperanzas latinoamericanas. El cambio ocurrido en el contexto internacional con el paso de la Guerra Fría a la "detente", constituyó otro factor del proceso de decadencia del sistema.

A. van Klaveren, op. cit., pp. 9-io.

"Por ejemplo, la estimación de la contribución de Chile a la guerra debido al precio tope arbitrariamente impuesto al cobre por los aliados, oscila entre los Us\$ 107 millones y los US $\$ 500$ millones. Ver Theodore Moran, Multinational Corporations and the Politics of Dependence: Copper in Chile (Princeton, N. J.: Princeton Univ. Press, 1977), p. 61.

"Ver Jerome Slater, "The Limits of Legitimization in International Organizations: The $\mathrm{OAS}^{\prime \prime}$ in Yale Ferguson (ed.), Contemporary Inter-American Relations (Englewood Cliffs, N. J.: Prentice Hall, 1973), PP: 204-226. 


\section{UN NUEVO CONTEXTO INTERNAGIONAL $Y$ NUEVOS} PROBLEMAS PARA EL SISTEMA

En una atmósfera más relajada, caracterizada por el surgimiento de nuevos poderes regionales, la recuperación de Europa Occidental y Japón, la decadencia progresiva de los antiguos bloques ideológicopolfticos, y la mejora de las relaciones entre Estados Unidos y la Unión Soviética y entre Estados Unidos y China, las naciones latinoamericanas trataron de maximizar los márgenes de autonomía en polf́tica exterior y defender sus propios intereses económicos, a menudo en conflicto directo con Estados Unidos. Durante este periodo muchas empresas norteamericanas en América Latina fueron nacionalizadas, ya que diversos países, grandes y pequeños, intentaron ejercer un control total sobre sus recursos naturales nacionales; paralelamente, los países de la región buscaron nuevos esquemas de integración como, por ejemplo, el Pacto Andino creado en 1969.

Uno de los signos más importantes del cambio que estaba ocurriendo en las relaciones Estados Unidos-América Latina, fue la entrega formal al Presidente Richard Nixon, por parte del Ministro de Relaciones Exteriores de Chile, Gabriel Valdés, en junio de 1969, del "Consenso de Viña del Mar" elaborado dentro del contexto de Ja cecla (Gomisión Especial para la Coordinación Latinoamericana). El documento afirmaba que existía "una profunda crisis en los hechos e instituciones del sistema interamericano", que los intereses de América Latina no eran idénticos a los de Washington (por el contrario, afirmaba el documento, mostraban la tendencia a ser "progresivamente contradictorios"); y que América Latina estaba entregando más de lo que recibía de los Estados Unidos. El "Consenso de Viña" provocó gran irritación en la Casa Blanca, y llevó a un enfriamiento considerable de las relaciones entre Washington y los países líderes de esta iniciativa latinoamericana?.

Durante la década del 70, nuevas tensiones se desarrollaron entre-Estados Unidos y América Latina. El descontento latinoamericano con el sistema interamericano, llevó a reformas concretas de la OEA. Así, en la reunión de San José en 1975, las naciones latinoamericanas introdujeron la noción de "seguridad económica colectiva", como parte de las conversaciones hemisféricas sobre asuntos estratégicos, pese a que Estados Unidos se opuso firmemente a esta expansión del concepto de seguridad. En esta misma conferencia de 1975, se modificó el Tratado de Río; entre otros puntos, se permitió el levantamiento, mediante mayoría simple en lugar de la votación de dos tercios, de las sanciones establecidas en el Tratado, facilitando

'Sobre este tema; ver Heraldo Muñoz y Carlos Portales, Estados Unidos y Chile: Una Relacion Dificil. (Por aparecer, 1987). 
de esta manera un acercamiento con Guba. Ese mismo año se formó el sELA (Sistema Económico Latinoamericano), como una organización regional de coordinación para asuntos económicos, que incluyó a Cuba y excluyó a Estados Unidos.

En el plano militar, la ayuda a la región proveniente de Washington declinó notablemente, debido a variadas razones relacionadas con cambios en el medio internacional y en la política interna norteamericana. Como consecuencia, algunos países latinoamericanos, particularmente aquellos gobernados por regímenes militares, acudieron a nuevos proveedores de armas y desarrollaron industrias bélicas locales, $y$, en algunos casos, sus propias perspectivas geopolíticas más allá de la "Doctrina de Seguridad Nacional". Es más, varias instituciones pertenecientes al sistema militar interamericano fueron eliminadas o seriamente cuestionadas por los latinoamericanos ${ }^{s}$. Durante la presidencia de James Carter, quien enfatizó la promoción de los derechos humanos en América Latina, las relaciones entre Washington y los entonces abundantes regimenes militares latinoamericanos, se deterioraron aún más. En resumen, hacia fines de la década del 70, Estados Unidos ya no controlaba la región como lo hiciera durante la segunda mitad de la década del 40, cuando se establecieron el Tratado de Rio y la oEA.

En notorio contraste con el caso dominicano de 1965, en junio de 1979, los Ministros de Relaciones Exteriores en la OEA rechazaron una proposición de Estados Unidos tendiente a crear una misión especial de la organización con el fin de negociar una transición en Nicaragua, como también rechazaron la idea de mandar una "fuerza de paz multilateral" para restablecer el orden en Managua. En lugar de ello, la oEA apoyó una moción del Grupo Andino que estimuló la victoria Sandinista sobre la dictadura de Somoza. No resultó extraño entonces que, cuando el gobierno de Reagan decidió invadir Grenada en 1983, la Casa Blanca haya buscado legitimidad v una "imagen de acción colectiva" en la oxcs (Organización de los Éstados del Caribe Oriental) y no en la OEA. Sin embargo, el tema fue analizado en la OEA, donde una amplia mayoría condenó la intervención y recomendó el retiro de las tropas estadounidenses de la Isla ${ }^{9}$.

Sin embargo, y más aún que en el caso de Grenada, la doctrina de seguridad colectiva imperante y el sistema de seguridad inter-

ser Child, op. cit., pp. 197-236.

${ }^{\circ} \mathrm{La}$ invasión de Grenada fue apoyada por los pafses del Caribe, que colaboraron en ella, pero ésta hizo más profundas las diferencias dentro de CARIcom y las existentes entre el Caribe y América Latina. Ver Committee on Foreign Affairs, U.s. House of Representatives, The English-Speaking Caribbean: Current Conditions and Implications for U.s. Policy. (Washington D. C., U.s. Goverment Printing Office, 1985). 
americano recibieron un golpe fatal durante la guerra de las $\mathrm{Mal}$ vinas entre Argentina y Gran Bretaña. A pesar de que la guerra no llegó a desmantelar el sistema y no significó una ruptura definitiva de las relaciones Estados Unidos-América Latina, sí demostró la total inefectividad del sistema como instrumento de seguridad colectiva. Como ha observado Connell-Smith, la oea fue incapaz de resolver un grave conflicto en el subcontinente, ni tampoco respaldó verdaderamente a uno de sus miembros que se encontraba en guerra con una potencia extracontinental ${ }^{10}$. Específicamente, provocó severos daños al Tratado de Río, reforzando el convencimiento de los estados latinoamericanos de que definitivamente su seguridad no podría ser dejada en las manos de Washington y que, por lo tanto, era esencial contar con un nivel adecuado de autosuficiencia militar.

Por último, el sistema de seguridad interamericano ha estado absolutamente ausente del conflicto centroamericano, pese a la patente violación a los Artículos 18, 19 y 20 de la Carta de la oEA, que prohíbe toda forma de intervención por parte de cualquier estado miembro contra otro gobierno de la organización, como es el caso de la guerra "no oficial" del gobierno de Reagan contra Nicaragua. Sin embargo, y a diferencia de lo ocurrido en el pasado, los países latinoamericanos han tratado de llenar el vacío dejado por la inacción del sistema interamericano, a través del mecanismo flexible del Grupo Contadora, cuyo propósito es contrarrestar el enfoque militar de Washington frente a la crisis, con proposiciones concretas para una solución pacífica y negociada.

Más allá del Grupo Contadora, la declaración conjunta de los presidentes de México y Brasil en mayo de 1983, en la que ambos países se oponen a la intervención externa en América Central, fue muy sintomática del sentimiento generalizado en América Latina en contra de la resurreción que Reagan ha hecho de las prácticas del "Gran Garrote". En este mismo tenor, en una reunión especial del SELA, se aprobó unánimemente una resolución que condena el boicot económico de Estados Unidos a Nicaragua, impuesto en mayo de 1985. En todo caso, por un lado Estados Unidos, debido a sus propios motivos, y por otro América Latina, según sus razones, han llegado a la conclusión de que el sistema interamericano debe ser ignorado cuando se trata de asuntos verdaderamente importantes tales como la crisis en América Central o la deuda externa dt la región.

\section{LA CONTRADICGIÓN ESENCIAL DEL SISTEMA: SEGURIDAD PARA}

Estados Unidos, INSEgURIDAd PaRA AmÉriga LatiNa

En las páginas anteriores hemos identificado los principales factores

${ }^{10}$ Gordon Connell-Smith, "The OAS and the Falklands Conflict", The World Today, vol. 38, No 9, septiembre 1982, pp. 340-347. 
que explican la fragmentación y decadencia progresiva del sistema interamericano de seguridad. En última instancia, todos estos factores convergen hacia una tensión fundamental y permanente; el sistema ha servido los intereses de seguridad de Estados Unidos, ig. norando aquellos de América Latina. En otras palabras, el sistema ha contribuido a la seguridad de Estados Unidos, en desmedro de la seguridad de América Latina ${ }^{11}$.

Las naciones latinoamericanas también tienen intereses específicos de seguridad que defender; pero, éstos han sido tradicionalmente postergados o jgnorados por Estados Unidos. Es más, la defensa de los intereses de Estados Unidos dentro del marco interamericano, se ha llevado a cabo en territorio de América Latina y el Caribe, trayendo a menudo como consecuencia la confrontación armada, la muerte de población civil, el desorden económico y el armamentismo a los países de la región.

La seguridad, desde la perspectiva latinoamericana, implica obviamente en un nivel fundamental, el elemento clásico de continuidad de la nación e integridad del territorio. Pero, como ya hemos sugerido, la seguridad de la región también implica seguridad política: es decir, autodeterminación efectiva y no intervención en los asuntos internos de los países del área. Por Io tanto, en este sentido, la seguridad de América Latina requiere que Estados U̇nidos: primero, se abstenga de intervenir en los asuntos internos de las naciones latinoamericanas y, segundo, inicie un proceso de "desintervencion" respecto a conductas y patrones que Washington ha ido creando con los años en concordancia con la "Doctrina de la Seguridad Nacional" y los criterios de contra-insurgencia.

La seguridad en América Latina también incorpora un componente socioeconómico vital: la capacidad interna para resolver problemas críticos de tipo social, económico y político de una manera igualitaria y democrática. Estos problemas domésticos no deben ser confundidos con asuntos de seguridad externa y deben ser enfrentados sin la interferencia de actores foráneos. Sin embargo, Washington ha considerado tradicionalmente el cambio social como una amenaza potencial a sus intereses de seguridad externa, mezclando de este modo las esferas externas e internas de la seguridad en América Latina. Irónicamente, entonces, el tipo de actitud y conducta de Estados Unidos que tiende a establecer una ecuación entre las transformaciones sociales y la amenaza externa (soviética), en última instancia constituye una amenaza para los intereses latinoamerica-

\footnotetext{
${ }^{11}$ En esta materia, mis comentarios se basan en la discusión sostenida durante el Seminario "Las Relaciones América Latina-Estados Unidos en la Segunda Mitad de los 80", organizado por el Instituto de Estudios de los Estados Unidos, CIDE, Ciudad de México, septiembre 1984, en el que participaron este autor y varios otros colegas latinoamericanos.
} 
nos; especificamente, representa un obstáculo para el cambio socioeconómico y político.

La preocupación de Estados Unidos por el desarrollo social y económico de la región ha sido esporádico y generalmente ha surgido cuando una crisis de seguridad necesitó de la solidaridad de América Latina con Washington. Este fue el caso de Ia Alianza para el Progreso, después de la revolución cubana; también fue el caso de la iniciativa de la Cuenca del Caribe anunciada por Reagan en 1982, y de las proposiciones de la Comisión Kissinger para América Central, difundidas a comienzos de 1984. En efecto, estos proyectos del gobierno republicano no estuvieron estimulados por una preocupación ilustrada acerca de las graves condiciones sociales y económicas imperantes en América Central y el Caribe, sino por una necesidad de complementar una política militar con un ofrecimiento de cooperación económica que hiciera más aceptable dicha política. La principal falla de estas iniciativas es su relación directa con los intereses de defensa de Estados Unidos.

A los ojos de Washington, el "status quo" tiende a ser considerado como el equivalente de "estabilidad política" $y$, a veces, incluso de democracia; de ahí que, los movimientos populares en favor del cambio y la democratización, generalmente producen desconfianza en Estados Unidos. Desde el punto de vista latinoamericano, sin embargo, la mera instalación de instituciones de democracia liliberal en la región no basta para garantizar el orden democrático, a menos que la democracia político-institucional vaya acompañada, simultáneamente, por profundas transformaciones socioeconómicas que la apoyen. Hay, empero, algunas esperanzas en este sentido, como puede concluirse del amplio consenso que surgió en el "Diálogo Interamericano" en favor de la proposición de que los cambios en América Latina y el Caribe no necesariamente constituyen una amenaza automática a la seguridad nacional de los Estados Unidos ${ }^{12}$.

En resumen, Estados Unidos ha subrayado consistentemente la dimensión estratégico-militar del sistema interamericano de seguridad; a la inversa, los países latinoamericanos, a pesar de que no niegan los aspectos militares de la relación, han enfatizado los problemas económicos y sociales, luchando por obtener mejores condi-

${ }^{1}$ El Diálogo Interamericano, que congregó a distinguidos ciudadanos privados de Estados Unidos y América Latina y el Caribe, afirma, en su primer documento, que es conveniente que los conflictos Este-Oeste permanezcan fuera de la región y que este propósito no se ve favorecido "si Estados Unidos se opone a los cambios en la región simplemente porque disminuyen la influencia de Estados Unidos y por lo tanto ellos son percibidos como ventajosos para Cuba Y la Unión Soviética, a menos que estén claramente relacionados con asuntos básicos de seguridad". (Ver Diálogo Interamericano, Las Américas en una Encrucijada. (Washington D. G.: Woodrow Wilson Center, abril de 1983, p. 42). 
ciones en los lazos comerciales, financieros y de inversión con Estados Unidos. La posición norteamericana siempre ha terminado por prevalecer, debido a la incapacidad de América Latina de responder con una posición común y unificada, o debido al hecho que, en momentos de crisis, la demanda de solidaridad hemisférica de Washington ha ido acompañada por una preocupación temporal por los problemas sociales y económicos de la región, preocupación que desaparece tan pronto como la meta de seguridad de corto plazo ha sido alcanzada.

La falta de comunicación entre Estados Unidos y América Ĺatina, en lo que respecta a la seguridad hemisférica, encuentra su mejor demostración en el debate sobre la deuda externa. Para el gobierno de Reagan, la deuda externa de América Latina puede resolverse, en esencia, mediante el estímulo de la inversión extranjera privada y el aumento de las exportaciones ${ }^{13}$. En contraste, para los países latinoamericanos, el problema de la deuda es la más grave amenaza a la seguridad que debe enfrentar hoy la región y, por consiguiente, han instado a los países acreedores a que participen en un esfuerzo por encontrar una respuesta común a un problema que no puede ser resuelto en términos puramente económicos. Es importante señalar que las naciones latinoamericanas han optado por mantener el problema de la deuda fuera del sistema interamericano y han creado, en cambio, mecanismos ad-hoc para su discusión, como es el caso del Consenso de Cartagena.

\section{Algunas conclustones Y SUGERENGLAS SOBRE LA SEGURIDAD COLECTIVA}

El sistema interamericano ha fracasado como esquema de seguridad hemisférica: Hoy en día, los países latinoamericanos consideran que hay mejores maneras de proteger su seguridad que la partic:pación en una alianza hemisférica rígida, como aquella establecida a fines de la década del 40 por Estados Unidos. Sin embargo, esto no quiere decir que todo el sistema interamericano sea inútil. Por ejemplo, la oEA, la principal institución del sistema, ha jugado un papel positivo durante los últimos años en la protección de los derechos humanos en América Latina, a través de su comisión ad-hoc, y, en forma excepcional, ha contribuido a la solución de disputas entre paises miembros ${ }^{14}$. Es más, es necesario mantener un vínculo perma-

\footnotetext{
${ }^{2}$ El Plan Baker anunciado por los Estados Unidos en octubre de 1985 en la reunión conjunta del FMr y el Banco Mundial en Seúl, representó un leve cambio hacia una solución política del problema de la deuda de las naciones del Tercer Mundo. Sin embargo, en América Latina, el Plan Baker es considerado como totalmente insuficiente, dada la envergadura del peso de la deuda.

${ }^{14} \mathrm{EI}$ ejemplo clásico es la exitosa mediación de la OEA en la guerra entre Honduras' y E1 Salvador en 1969. Sin embargo, la evidencia demuestra que "vir-
} 
nente entre Estados'Unidos y América Latina, para lo cual podría ser muy útil un sistema interamericano adaptado, rediseñado en forma de foro para el intercambio hemisférico pero no como una alianza continental.

Gon todo, es necesario contar con alguna instancia de seguridad regional para enfrentar los diversos tipos de conflictos que inevitablemente ocurrirán en América Latina debido a rivalidades tradicio. nales, disputas de poder u otras variables. En Latinoamérica se acepta cada vez más la idea de que un sistema alternativo de seguridad debe implicar un acuerdo político entre los poderes mundiales en favor de un esfuerzo de cooperación regional para evitar conflictos, junto con un proceso de desmilitarización y desarme dentro de los países de América Latina y el Caribe.

El principal propósito de un sistema de seguridad alternativo de este tipo, y que se conoce como la opción "Zona de Paz"15, sería separar a América Latina y el Caribe de la lógica del conflicto EsteOeste y concentrar las energías nacionales en los procesos de desarrollo interno. Esto plantea la pregunta de "seguridad para quien", prefiriéndose la seguridad de los sectores populares o de las grandes mayorías de nuestras naciones. Paralelamente, ello significa que los problemas de seguridad de la región son más económicos, sociales y políticos, que militares o estratégicos. En todo caso, el esquema de seguridad escogido debiera tener en cuenta no solamente los intereses de seguridad de América Latina y el Garibe, sino también los intereses y percepciones de amenaza a la seguridad nacional de Estados Unidos. De ahí que, como ha indicado Portales ${ }^{16}$, la proposición de Zona de Paz debiera implicar:

1. El retiro de todas las bases militares y tropas externas a la región. Obviamente, este sería un proceso gradual; pero, en última intancia, debería incluir la presencia militar de Estados Unidos en Panamá, Honduras y otros países; el despliegue militar soviético en Cuba, y la presencia británica en las Malvinas;

2. La desnuclearización efectiva de la región a través de la implementación real del Tratado de Tlatelolco;

3. Un plan-para controlar el gasto en defensa y permitir el avance hacia el desarme en la región, en los términos propuestos

tualmente todos los esfuerzos exitosos de mediación del Sistema Interamericano tuvieron éxito debido a instancias ad-hoc utilizadas por personalidades fuertes". (Ver Ronald Scheman Y John W. Ford, "The Organization of American States as Mediator", en S. Touval y I. W. Zartman (eds.), International Mediation in Theory and Practice. (Boulder: Westview Press. 1985, pp. 197-231).

${ }^{15} \mathrm{La}$ idea de establecer Zonas de Paz en diversas regiones del mundo fue introducida por primera vez y aprobada en una sesión de la onv en junio de 1978.

${ }^{16}$ Carlos Portales, "Zona de Paz: una Alternativa a los Desafíos Estratégícos de América Latina", mimeo., FLACSO-Santiago. Noviembre 1984, pp. 23-24. 
recientemente por el Presidente peruano, Alan Garcia ${ }^{17}$. Un buen comienzo-sería un acuerdo de no-introducción de nuevo armamento sofisticado en el área;

4. La total aceptación de este nuevo estatuto regional por parte de las grandes potencias.

La implementación de una proposición tan ambiciosa requeriria de negociaciones bilaterales y subregionales al estilo del Grupo Contadora. Sería poco realista intentar negociaciones a nivel regional, especialmente teniendo en consideración el conflicto centroamericano; por lo tanto, América del Sur podría ser el foco inmediato para una eventual Zona de Paz Latinoamericana.

Un paso básico en la dirección del control de armas y la paź, sería la implementación de "medidas para incrementar la confianza mutua". Este concepto, vinculado con el acuerdo de Helsinki de 1975, se refiere a aquellas acciones que pueden "reducir los riesgos de conflicto armado, equivocaciones o errores de cálculo en las actividades militares que pudieran producir sospecha o temor, especialmente en situaciones en que los estados implicados carecen de la información correspondiente y adecuada"18. El Grupo Contadora ha propuesto varias ideas interesantes al respecto, tales como: treinta días de notificación previos a cualquier maniobra militar en un radio de treinta kilómetros de las fronteras; invitación a observadores de países vecinos; prohibición de operaciones militares internacionales en los territorios involucrados; eliminación de la transferencia de armas para fuerzas irregulares; reducción de Ios consejeros militares externos en las naciones que participen; comisiones mixtas de seguridad, y un sistema regional de comunicaciones para mantener en contacto a los gobiernos del área.

Obviamente, no es nada fácil llevar a la práctica lo que acabamos de describir. Establecer una "Zona de Paz" en América Latina es, por decir lo menos, una tarea muy difícil de realizar, ya que requeriría de un compromiso constante de parte de los países implicados directamente, y también de la cooperación de las superpotencias. Por otro lado, no existe otra alternativa clara a la vista. $\mathrm{La}$ creación de una Zona de Paz en la región puede ser, a fin dè cuentas, una utopía. Sin embargo, la continuación del actual sistema interamericano de seguridad colectiva es aún más irreal, ya que su inoperancia ha quedado bastante demostrada.

ITEI texto de la proposición de desarme de Alan García se encuentra en el libro de Hugo Palma, América Latina: Limitación de Armamentos y Desarme en la Región (Lima: CEPEI, 1986), pp. 202-204.

1sJozef Goldblat, citado por Victor Millán: "Mecanismos para Aumentar Ia Confianza Mutua en América Latina", trabajo presentado en la Conferencia "Seguridad Regional y Paz en América Latina", Instituto de Estudios Internacionales, Uniyersidad de Chile, Viña del Mar. 12 al 22 de noviembre de 1984 , p. 1 . 